\title{
EXACT ANALYTICAL SOLUTION FOR A PIE-SHAPED WEDGE THICK PLATE UNDER OSCILLATING LOAD
}

Received: date / Accepted: date

\begin{abstract}
A finite elastic wedge shaped thick plate is considered. One of the faces is based on a rigid base, the other is exposed to a dynamic oscillating load through an absolutely rigid overlay. On the side faces conditions of sliding contact are fulfilled, at its end the stresses are given. The solution is based on a special linear transformation of a Lame's equations and application of the vector integral transformations' method. The proposed approach leads to a one-dimensional vector boundary value problem for which an exact solution is constructed. The analysis of the eigenfrequency values distribution and estimation of the edge resonance frequency are done. An analogous problem is solved for the case when at the lower wedge-plate's face the sliding conditions are given. In order to establish the possibility of separation of the lower base, a comparison of the obtained values of the stress on the bottom plate with the stresses arising in the analogous formulation of the static problem for wedge plate considering its own weight was worked out.
\end{abstract}

Keywords wedge shaped thick plate · integral transformation · one-dimensional vector boundary problem · exact solution

\section{Introduction}

Dynamic elasticity problems for wedge-shaped bodies are of obvious interest in both theory and applications. Wedge-shaped three dimensional structures have the wide range of industrial needs in aircraft construction, reinforced concrete, advanced composite materials, etc. They are one of the most relevant models in many engineering applications such as civil, military and marine structures also. The wedge shaped constructions are widely present in the geological bodies, and the mechanics of the wedge-shaped blocks with basal boundaries and so are of great interest and importance [1]. Such constructions are used as the elements of transport vehicles and engines, military, aerospace engineering, etc. The influence of different strikes and bumps leads to the onset of three-dimensional dynamic stress state in pie-wedged bodies. The theories of shells, shafts and plates are applied usually for to solve the corresponding boundary valued problems in these situations. The theory of torsion and bending of shafts, the generalized plane stress state and plane deformation describe a dynamic stress field. However, all these theories are the approaches in some way, the applicability of which in some cases is executed approximately. For trustworthy positive information on the extreme values of stress near the

O. Menshykov, M. Menshykova

School of Engineering, College of Physical Sciences, Fraser Noble Building, Kings College, Aberdeen, AB24 3UE, Scotland, United Kingdom

N. Vaysfeld

Odesa Mechnikov University, Institute of Mathematics, Economics and Mechanics, str. Dvoryanska, 2, 65082, Odesa, Ukraine E-mail: vaysfeld@onu.edu.ua 
areas of loading influence, at the zones of the rapidly changing of a construction's geometry or near the edges of a body one should use the methods and results of the spatial problems analytical solutions.

Due to the practical importance the analytical solving methods that can provide accurate results are vital in the understanding of a three-dimensional constructions' dynamic characteristics and have thus received much attention from the researchers over the past twenty years. Three-dimensional analytical solutions give an advantage for direct comparisons with the results of more simple plate's theories, an analytical exact solution helps to compare results with other numerical methods and evaluation with experimental results. Problems for the oscillatory processes are discussed and studied in [2]-[10], in which detailed and extensive approaches were worked out and methods created for dynamical analysis of elastic bodies under oscillation loading. This mathematical apparatus was applied successfully to solve the complicated dynamic problem for bodies of rectangular, cylindrical and spherical shapes, circular disks and plates [11]-[20].

It should be noted that the problem for the wedge-shaped bodies in view of the complexity of its geometry has been studied much less. The mathematical difficulties of three-dimensional pie-shaped wedge problems are sufficiently greater than their two-dimensional analogues. The estimation of the stress field in a pie-shaped body was until recently a serious problem for researchers. Nevertheless, some important results have been achieved in static and dynamic statements of elasticity problems for a pie-shaped construction.

Three-dimensional asymptotic singular stress fields near the front of a wedge are presented in [21]. This investigation is devoted to the establishment of rigorous conditions as to whether the presence of wedge or end face stress singularity, which depends on the prescribed boundary condition, can validate or invalidate the heuristic assumption implicit in SaintVenant's principle. This is accomplished by applying a general approach to the solution of canonically singular problems, based on the concept of the proper boundary-value problem, the theorem of homogeneous solutions, and classification of boundary value problems (BVP) of three-dimensional elasticity theory into class S (SaintVenant) or class N (non-SaintVenant).

In a static statement the problems for pie-shaped wedge plates were considered in [22]-[23]. Fundamental solutions for a three-dimensional wedge are used to investigate problems of a thin, rigid, elliptic inclusion in a wedge [22]. A regular asymptotic form is employed which has previously been used in contact problems for a wedge and in problems of a crack in a wedge in the case of an elliptic shape of the contact region or crack. The method is effective in the case of an inclusion which is sufficiently distant from an edge of the wedge when the known exact solution for the space can be taken as the zero-th approximation. A numerical analysis and comparison of different characteristics of wedge problems is carried out.

The exact solution of the first main elasticity problem for the wedge-shaped thick infinite elastic plate was constructed in [23]. There the equilibrium equations were solved directly without use of intermediary harmonic or byharmonic functions. The constructed solution fully describes the stress state of the infinite spatial pie-shaped elastic construction. On the base of the proposed approach, the more complicated mixed boundaryvalue elasticity problem for an infinite wedge-shaped plate with regard to its proper weight was solved [23]. The exact solution was constructed and stress values were analyzed in dependence of the proper weight of the pie-shaped construction.

The dynamic effects of a wedge-shaped plate were studied for steady harmonic oscillations in the composite edge-like area in [24], where the authors proposed a special investigation method to solve the boundary-value problem. The steady vibrations of circular and annular plates were investigated numerically in [20], [26].

The special investigation method is applied to analyze the stress state of a composite wedge-like body [27].

In [28] the authors proposed the analytical method to solve the problem of free vibrations of thick sectorial plates with simply supported radial edges. An analytical method to obtain an exact solution of the three-dimensional problem of the wedge plate's free vibrations with some special boundary conditions on its surface, was presented in [29]. A unified method for free vibration analysis of circular, annular and sector plates with arbitrary boundary conditions is presented in [30]. In [31] the method of constructing an exact solution's for a pie-shaped wedge thick plate under an oscillating dynamical load is given.

One of the important directions in studying the oscillatory processes is the determination of the elastic bodies' eigenfrequencies. This phenomenon was studied in detail and comprehensively for finite 
elastic cylinders, disks and rectangles [32]-[35]. The study of eigenfrequencies for a wedge shaped plate apparently has not previously been conducted. Also useful is the investigation of an edge resonance phenomenon, by which one means the eigen oscillations with significant localized zones of an increased intensity of dynamic stress state around certain parts of the boundary surfaces. This phenomenon was studied in detail and comprehensively for isotropic and transversely isotropic bodies of canonical form, but studies for elastic wedge shaped bodies in the literature are not mentioned.

The conducted analyses of the researches of dynamical stress state of an elastic pie-shaped wedge under oscillating load shows that the question of the exact estimations of its wave field is still open. Analytical solutions of this problem are unavailable, despite the necessity for it for many engineering applications.

In the proposed work an exact solution of the dynamic problem for a finite wedge plate is derived. The eigenfrequencies' spectrum is analysed and the edge resonance's frequencies are detected.

\section{The problem statement and its reduction to a one-dimensional vector boundary problem}

The elastic wedge shaped thick plate (Fig. 1) is defined in the cylindrical coordinate system as

$$
0 \leq r \leq a, 0 \leq \varphi \leq \Upsilon, 0 \leq z \leq h .
$$

A face plate $z=h$ (the lower face) is considered to be based on a rigid smooth base

$$
u_{z}(r, \varphi, h, t)=0, \tau_{z r}(r, \varphi, h, t)=0, \tau_{z \varphi}(r, \varphi, h, t)=0
$$

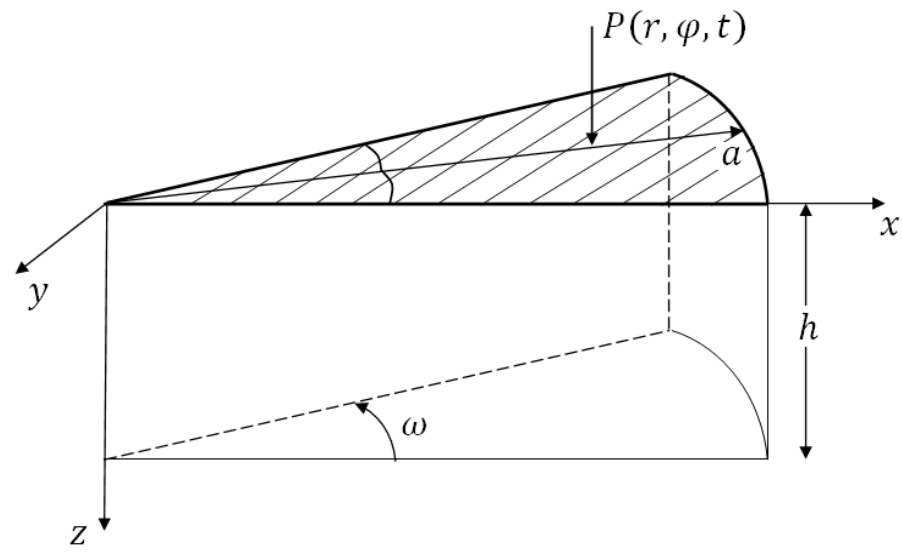

Fig. 1 Wedge shaped plate

In smooth contact with the face $z=0$ (top face) is an absolutely rigid plate of known mass $m$, which has the same form as the upper face of the plate. The concentrated force $P(t) P \cos \tilde{\omega} t(\tilde{\omega}$ is the load's frequency) is applied to the rigid plate through the line $0<r<a, \varphi=\Upsilon / 2$ at a distance $l$ from the vertex of the wedge. Under force's influence the points of the upper bound are shifted at the value $\delta+\operatorname{Ar} \cos \varphi$, and the conditions on the face $z=0$ can be written as

$$
u_{z}(r, \varphi, 0, t)=(\delta+A r \cos \varphi) \cos \tilde{\omega} t, \tau_{z r}(r, \varphi, 0, t)=0, \tau_{z \varphi}(r, \varphi, 0, t)=0
$$

The unknown constants $\delta, A$ are determined from the rigid plate's movement equations, which on the basis of the D'Alamber's principle are written as:

$$
\begin{gathered}
-P(t)+\int_{-\pi}^{\pi} \int_{0}^{a} \sigma_{z}(r, \varphi, h, t) r d r d \varphi-\left.m \frac{\partial^{2} u_{z}}{\partial t^{2}}\right|_{z=h}=0 \\
-l P(t)+\int_{-\pi}^{\pi} \int_{0}^{a} \sigma_{z}(r, \varphi, h, t) r^{2} \cos \varphi d r d \varphi-\left.\frac{2 m a \sin ^{2} \Upsilon}{3 \Upsilon} \frac{\partial^{2} u_{z}}{\partial t^{2}}\right|_{z=h}=0
\end{gathered}
$$


The conditions of smooth contact are fulfilled at the faces $\varphi=0$ and $\varphi=\Upsilon$

$$
\left.u_{\varphi}(r, \varphi, z, t)\right|_{\varphi=0, \Upsilon}=0,\left.\tau_{\varphi z}(r, \varphi, z, t)\right|_{\varphi=0, \Upsilon}=0,\left.\tau_{\varphi r}(r, \varphi, z, t)\right|_{\varphi=0, \Upsilon}=0
$$

On the face $r=a$ the conditions

$$
\sigma_{r}(a, \varphi, z, t)=0, \tau_{r \varphi}(a, \varphi, z, t)=0, \tau_{r z}(a, \varphi, z, t)=0
$$

are assumed (elasticity first fundamental problem).

It is required to find the solution of the problem satisfying the boundary conditions (2), (3), (5), (6) and the movement equations for the displacements $u_{r}(r, \varphi, z, t)=u(r, \varphi, z) \cos \tilde{\omega} t, u_{\varphi}(r, \varphi, z, t)=$ $v(r, \varphi, z) \cos \tilde{\omega} t, u_{z}(r, \varphi, z, t)=w(r, \varphi, z) \cos \tilde{\omega} t$ (one assumes that all of the functions are time-dependent according to the law $f(r, \varphi, z, t)=f(r, \varphi, z) \cos \tilde{\omega} t)$

$$
\begin{gathered}
\frac{\left(r u^{\prime}\right)^{\prime}}{r}+\frac{u^{\prime}}{r^{2}}+u^{\prime \prime}+q^{2} u-\frac{u}{r^{2}}-\frac{2 v}{r^{2}}+\mu_{0}\left[\frac{\left(r u^{\prime}\right)^{\prime}}{r}-\frac{u}{r^{2}}-\frac{v^{\prime}}{r^{2}}+\frac{v^{\prime}}{r}+w^{\prime}\right]=0, \\
\frac{\left(r v^{\prime}\right)^{\prime}}{r}+\frac{v^{\prime}}{r^{2}}+v^{\prime \prime}-\frac{v}{r^{2}}+q^{2} v+\frac{2 u}{r^{2}}+\frac{\mu_{0}}{2}\left[u^{\prime}+\frac{u}{r}+\frac{v}{r}+w^{\prime}\right]=0 \\
\frac{\left(r w^{\prime}\right)^{\prime}}{r}+\frac{w^{\prime}}{r^{2}}+w^{\prime \prime}+q^{2} w \mu_{0}\left[u^{\prime}+\frac{u}{r}+\frac{v^{\prime}}{r}+w^{\prime}\right]=0
\end{gathered}
$$

Here $q^{2}=\frac{\tilde{\omega}^{2} \rho}{G}=\tilde{\omega}^{2} c^{2}, \mu_{0}=(1-2 \nu)^{-1}, G, \rho, \nu$ are a shear modulus, a density and a Poisson's ratio correspondently, a stroke above a letter denotes the derivative with respect to the first variable, a point above a letter - to the second variable, a comma denotes the derivative with respect to the third variable (here and further a wave above a letter is omitted).

\section{The problem's reduction to the one-dimensional boundary problem}

With regard to change of variables, the boundary conditions will take the form

$$
\begin{aligned}
& u^{\prime}(r, \varphi, h)=0, v^{\prime}(r, \varphi, h)=0, w(r, \varphi, h)=0 \\
& u^{\prime}(r, \varphi, 0)=-\delta^{\prime}(r, \varphi), v^{\prime}(r, \varphi, 0)=-\frac{1}{r} \delta \cdot(r, \varphi), w(r, \varphi, 0)=\delta(r, \varphi) \\
& \left.u \cdot(r, \varphi, z)\right|_{\varphi=0, \Upsilon}=0,\left.v(r, \varphi, z)\right|_{\varphi=0, \Upsilon}=0,\left.w \cdot(r, \varphi, z)\right|_{\varphi=0, \Upsilon}=0 \\
& \begin{array}{c}
{\left.\left[(1-\nu) u^{\prime}+v\left(\frac{u}{r}+\frac{v}{r}+w^{\prime}\right)\right]\right|_{r=a}=0,} \\
{\left[\frac{u^{\cdot}-v}{r}+v^{\prime}\right]_{r=a}=0,\left.\left[w^{\prime}+u^{\prime}\right]\right|_{r=a}=0}
\end{array}
\end{aligned}
$$

One should apply the Fourier's transformation with regard to the variable $\varphi$ to the movement equations (7) and the boundary conditions (8-10)

$$
\begin{aligned}
& {\left[\begin{array}{c}
u_{n}(r, z) \\
w_{n}(r, z)
\end{array}\right]_{\Upsilon}=\int_{0}^{\Upsilon} \cos \alpha_{n} \varphi\left[\begin{array}{c}
u(r, \varphi, z), \\
w(r, \varphi, z)
\end{array}\right] d \varphi ; n=0,1,2, \ldots} \\
& v_{n}(r, z)=\int_{0} \sin \alpha_{n} \varphi v(r, \varphi, z) d \varphi ; n=1,2,3, \ldots ; \alpha_{n}=\frac{\pi n}{\Upsilon}
\end{aligned}
$$

To the transformed equations and the boundary conditions (10) the Fourier's transformation with regard to the variable $z$ was applied as

$$
\begin{gathered}
{\left[\begin{array}{c}
u_{n k}(r) \\
v_{n k}(r)
\end{array}\right]=\int_{0}^{h} \cos \beta_{k} z\left[\begin{array}{c}
u_{n}(r, z), \\
v_{n}(r, z)
\end{array}\right] d z ; \beta_{k}=\frac{\pi k}{h}, n=0,1,2, \ldots} \\
w_{n k}(r)=\int_{0}^{h} \sin \beta_{k} z w_{n}(r, z) d z ; k=1,2,3, \ldots
\end{gathered}
$$


One has to use it with the transformed boundary conditions (8), and finally in the domain of the integral transformations (11), (12) the movement equations and the boundary conditions will take the form

$$
\begin{gathered}
\frac{\left(r u_{n k}^{\prime}\right)^{\prime}}{r}-\alpha^{2} \frac{u_{n k}}{r^{2}}-\beta^{2} u_{n k}+q^{2} u_{n k}-\frac{2 \alpha v_{n k}}{r^{2}}+ \\
+\mu_{0}\left[\frac{\left(r u_{n k}^{\prime}\right)^{\prime}}{r}-\frac{u_{n k}}{r^{2}}-\frac{\alpha v_{n k}^{\prime}}{r^{2}}+\beta w^{\prime}\right]=\left(\mu_{0}-1\right) \delta_{n}^{\prime}(r) \\
(n=0,1,2, \ldots ; k=1,2,3, \ldots) \\
\quad \frac{\left(r v_{n k}^{\prime}\right)^{\prime}}{r}-\frac{\alpha^{2} v_{n k}}{r^{2}}-\beta^{2} v_{n k}-\frac{v_{n k}}{r^{2}}+q^{2} v_{n k}- \\
-\frac{2 \alpha u_{n k}}{r^{2}}-\mu_{0} \frac{\alpha}{r}\left[u_{n k}^{\prime}+\frac{u_{n k}}{r}+\frac{\alpha v_{n k}}{r}+\beta w_{n k}\right]= \\
=\left(1-\mu_{0}\right) \alpha \frac{\delta_{n}(r)}{2}(n=0,1,2, \ldots ; k=1,2,3, \ldots) \\
\quad \frac{\left(r w_{n k}^{\prime}\right)^{\prime}}{r}-\alpha^{2} \frac{w_{n k}}{r^{2}}-b e t a^{2} w_{n k}+q^{2} w_{n k}- \\
\quad-\mu_{0} \beta\left[u_{n k}^{\prime}+\frac{u_{n k}}{r}+\alpha \frac{v_{n k}}{r}+\beta w_{n k}\right]= \\
=-\beta \mu_{*} \delta_{n}(r)(n=0,1,2, \ldots ; k=1,2,3, \ldots) \\
u_{n k}(a)+\bar{\mu}\left[a^{-1} u_{n k}(a)+\alpha v_{n k}(a)+\beta w_{n k}(a)\right]=\bar{\mu} \delta_{n}(a), \\
v_{n k}^{\prime}(a)-\alpha a^{-1} u_{n k}(a)-v_{n k}(a)=0, w_{n k}^{\prime}(a)-\beta u_{n k}(a)=0
\end{gathered}
$$

Here the notations $\alpha=\alpha_{n}, \beta=\beta_{k}, \mu_{*}=1+m_{0}, \bar{\mu}=\left(\mu_{0}-1\right) \mu_{*}^{-1}, \delta_{n}(r)=2(-1)^{n} A r \sin \Upsilon$, are introduced. At first it is useful to consider some particular cases of the proposed problem.

3.1 Solution of the one-dimensional vector boundary problem for the case $n=0, k \geq 1$

Let's state the vector boundary problem, taking into account that $v_{0 k}=0, k \geq 1$. The system (13) disintegrates on two equations. The vectors

$$
\begin{aligned}
& \mathbf{y}_{0 k}(r)=\left(\begin{array}{c}
u_{0 k}(r) \\
w_{0 k}(r)
\end{array}\right), \mathbf{f}_{0 k}(r)=\left(\begin{array}{c}
\bar{\mu} \delta_{0}^{\prime}(r) \\
-\mu_{*} \beta_{k} \delta_{0}(r)
\end{array}\right), \gamma(r)=\left(\begin{array}{c}
\bar{\mu} \delta_{0}(r) \\
0
\end{array}\right) \\
& \text { and the matrixes } \mathbf{L}_{0 k}=\left(\begin{array}{c}
D_{r}-r-2-\beta_{k}^{2} \mu_{*}^{-1}+q^{2} \mu_{*}^{-1}, \mu_{0} \mu_{*}^{-1} \beta_{k} \partial_{r} \\
-\mu_{0} \beta_{k}\left(\frac{\partial}{\partial r}+\frac{1}{r}\right), D_{r}-\beta_{k}^{2} \mu_{*}+q^{2}
\end{array}\right), \mathbf{U}_{0 k}=\left(\begin{array}{c}
\bar{\mu} a^{-1}+\partial_{r}, \bar{\mu} \beta \\
-\beta, 1
\end{array}\right),
\end{aligned}
$$

$D_{r}=r^{-1}\left(r f^{\prime}\right)^{\prime}, \partial_{r}=f^{\prime}$ are introduced to construct the vector boundary-value problem. The system (13) and transformed boundary conditions (14) are written in the form

$$
\begin{gathered}
\mathbf{L}_{0 k}\left(\mathbf{y}_{0 k}(r)\right)=\mathbf{f}_{0 k}(r), 0<r<a \\
\mathbf{U}_{0 k}\left(\mathbf{y}_{0 k}(r)\right)=\gamma, k \geq 1
\end{gathered}
$$

The solution of the inhomogeneous vector equation is derived as the superposition of the homogeneous equation's (13) general solution $\mathbf{y}_{0}(r)$ and the particular solution $\mathbf{y}_{* k}(r)$ of the inhomogeneous equation [36]. One must first solve the homogenous matrix equation $L_{0 k}(\mathbf{Y}(r))=0,0<r<a$ to construct $\mathbf{y}_{0}(r)[37]$.

The matrix $\mathbf{H}_{0 k}(r, s)$ is found for it. This matrix satisfies the equality $L_{0 k}\left(\mathbf{H}_{0 k}(r, s)\right)=-\mathbf{H}_{0 k}(r, s) \mathbf{M}_{0 k}(r, s), 0<$ $r<a$. One can be sure of it by the direct checking the matrix $\mathbf{H}_{0 k}(r, s)=\left(\begin{array}{c}J_{1}(r s), 0 \\ 0, J_{0}(r s)\end{array}\right)$ satisfies to this demand (the regularity in zero is guaranteed). The matrix $\mathbf{M}_{0 k}(r, s)$ has the form $\mathbf{M}_{0 k}(r, s)=\left(\begin{array}{c}s^{2}+\beta^{2} \mu_{*}^{-1}-q^{2} \mu_{*}^{-1}, \mu_{0} \mu_{*}^{-1} \beta s \\ \mu_{0} \beta s, s^{2}+\beta^{2} \mu_{*}^{-1}-q^{2}\end{array}\right)$.

The solution of the matrix equation is given by the formula [37] $\mathbf{Y}(r)=\frac{1}{2 \pi i} \oint_{C} \mathbf{H}_{0}(r, s) \mathbf{M}_{0 k}^{-1}(s) d s$, where $C$ is the closed contour, covering the poles $s_{i}, i=\overline{1,4}$ of the matrix $\mathbf{M}_{0 k}^{-1}(s)$.

$$
\begin{gathered}
\mathbf{M}_{0 k}^{-1}(s)= \\
=\frac{(2 \nu-1)^{-1}(2 \nu-2)^{-1}}{4}\left(\begin{array}{c}
\frac{\left(2(\nu-1) s^{2}+2(\nu-1)\left(\beta^{2}+q^{2}\right)\right)}{2(\nu-1)^{-1}}, \beta s(2 \nu-1) \\
2(\nu-1) \beta s, \frac{\left(2(\nu-1) s^{2}+2(\nu-1)\left(\beta^{2}+q^{2}\right)\right)}{2(\nu-1)^{-1}}
\end{array}\right) \\
\prod_{i=1}^{4}\left(s-s_{i}\right) \\
s_{1,2}= \pm \sqrt{q^{2}-\beta^{2}}, s_{3,4}= \pm \frac{\sqrt{\left(2 q^{2}(1-2 \nu)-4 \beta^{2}(1-\nu)\right)}}{2 \sqrt{(\nu-1)}}
\end{gathered}
$$


The contour $C$ can be taken as the contour, covering only one of these poles, for example, $s_{1}$. After the calculation of the residuals, the solution of the homogenous matrix equation is derived

$$
\begin{gathered}
\mathbf{Y}(r)=\left(\begin{array}{l}
Y_{11}, Y_{12} \\
\left.Y_{21}, Y_{22}\right)
\end{array}\right) \\
Y_{11}=\left\{\begin{array}{l}
\frac{(-\nu+1) \beta^{2} J_{1}\left(\sqrt{q^{2}-\beta^{2} r}\right)}{\sqrt{q^{2}-\beta^{2}}}, q>\beta, \\
\frac{(-\nu+1) \beta^{2} I_{1}\left(\sqrt{q^{2}-\beta^{2} r}\right)}{\sqrt{q^{2}-\beta^{2}}}, q<\beta,
\end{array}\right. \\
Y_{12}=\left\{\begin{array}{l}
\beta J_{1}\left(\sqrt{q^{2}-\beta^{2} r}\right), q>\beta, \\
\beta i I_{1}\left(\sqrt{q^{2}-\beta^{2} r}\right), q<\beta,
\end{array}\right. \\
Y_{21}=\left\{\begin{array}{l}
\beta(\nu-1) J_{0}\left(\sqrt{q^{2}-\beta^{2} r}\right), q>\beta, \\
\beta(\nu-1) I_{0}\left(\sqrt{q^{2}-\beta^{2} r}\right), q<\beta,
\end{array}\right. \\
Y_{22}=\left\{\begin{array}{l}
-\sqrt{q^{2}-\beta^{2}} J_{0}\left(\sqrt{q^{2}-\beta^{2} r}\right), q>\beta, \\
-i \sqrt{q^{2}-\beta^{2}} I_{0}\left(\sqrt{q^{2}-\beta^{2} r}\right), q<\beta .
\end{array}\right.
\end{gathered}
$$

here $J_{i}(x), I_{i}(x), i=0,1$ are the Bessel functions of the first kind and the corresponding modified Bessel functions [39]. As can be seen, the second column of the matrix in the case takes pure image values, that is the reason for providing the displacements' real value in this case (with regard of the known correspondences for the Bessel functions $\left.J_{0}(i z)=I_{0}(z), J_{1}(i z)=i I_{1}(z)[38]\right)$, the solution of the initial problem is constructed in the form

$$
\begin{gathered}
u_{0 k}(r)=C_{1} Y_{11}(r)+C_{2} Y_{12}(r), v_{0 k}(r)=C_{1} Y_{21}(r)+C_{2} Y_{22}(r), q>\beta \\
u_{0 k}(r)=C_{1} Y_{11}(r)+i C_{2} Y_{12}(r), v_{0 k}(r)=C_{1} Y_{21}(r)+i C_{2} Y_{22}(r), q<\beta
\end{gathered}
$$

where $C_{i}, i=1,2$, are the unknown real constants; $Y_{i j}, i, j=1,2$ are the elements of the matrix solution for the homogenous equation.

The particular solution of the inhomogeneous matrix equation is constructed with the use of the fundamental matrix of the equation. Let's define the vector equation (15) along the whole numerical axis. The right hand part of the equation is taken in the form $\mathbf{f}_{0 k}^{+}(r)=\left\{\begin{array}{c}\mathbf{f}_{0 k}(r), 0<r<a \\ 0, r>a\end{array}\right.$ for this. The integral Hankel transformation with the kernel $\mathbf{H}_{0 k}(r, s)$ is applied to the equation. One obtains the transformation of the particular solution $\mathbf{y}_{* k}(r)=\int_{0}^{\infty} s \mathbf{H}_{0}(\rho s) \mathbf{y}_{* k s} d s$ after applying the inverse formula to the correspondence $\mathbf{y}_{* k s}=-\mathbf{M}_{0 k}^{-1}(s) \int_{0}^{\infty} \rho \mathbf{H}_{0}(\rho s) \mathbf{f}_{0 k}^{+}(\rho) d \rho$.

As a result the formula is constructed in the form $\mathbf{y}_{* k}(r)=-\int_{0}^{a} \Phi_{0 k}(r, \rho) \mathbf{f}_{0 k}^{+}(\rho) d \rho$, where $\Phi_{0 k}(r, \rho)$ is the fundamental matrix $\Phi_{0 k}(r, \rho)=\int_{0}^{\infty} s \mathbf{H}_{0}(r, s) \mathbf{M}_{0 k}^{-1} \mathbf{H}_{0}(\rho, s) d s$. All elements of this matrix are expressed through the integrals of such form

$$
\int_{0}^{\infty} \frac{s^{m} J_{\mu}(r s) J_{\nu}(\rho s)}{\left(s^{2}+A^{2}\right)\left(s^{2}+B^{2}\right)} d s=\Omega_{\mu, \nu}^{m}
$$

where $m=0,1,2,3, A^{2}=\beta^{2}-q^{2}, B^{2}=\frac{\left(q^{2}(1-2 \nu)+2 \beta^{2}(\nu-1)\right)}{2(1-\nu)}$, which are reduced to the known table integrals [39]. The partial solution of the inhomogeneous equation is constructed. 
The displacements are given as

$$
\begin{gathered}
u_{* k}(r)=-\frac{2 \nu-2}{2 \nu-1} \bar{\mu} \int_{0}^{a} \rho \delta_{0}^{\prime}(\rho) \Omega_{1,1}^{1}(r, \rho) d \rho-\frac{2(\nu-2) \beta^{2}+(1-2 \nu) q^{2}}{(2 \nu-1)} \bar{\mu} \int_{0}^{a} \rho \delta_{0}^{\prime}(\rho) \Omega_{1,1}^{0}(r, \rho) d \rho \\
\quad+\frac{\beta \mu_{*}}{2(\nu-1)} \int_{0}^{a} \rho \delta_{0}(\rho) \Omega_{1,0}^{2}(r, \rho) d \rho, \\
w_{* k}(r)=-\frac{\beta \bar{\mu}}{(2 \nu-1)} \int_{0}^{a} \rho \delta_{0}^{\prime}(\rho) \Omega_{1,0}^{2}(r, \rho) d \rho-\mu_{*} \int_{0}^{a} \rho \delta_{0}(\rho) \Omega_{0,0}^{1}(r, \rho) d \rho- \\
-\frac{(2 \nu-1) \beta^{2}+(1-2 \nu) q^{2}}{2(\nu-1)} \mu_{*} \int_{0}^{a} \rho \delta_{0}(\rho) \Omega_{0,0}^{0}(r, \rho) d \rho .
\end{gathered}
$$

The solution of the initial problem $u_{k}(r)=u_{0 k}(r)+u_{* k}(r), w_{k}(r)=w_{0 k}(r)=w_{* k}(r)$ will finally be derived, if the unknown constants $C_{i}, i=1,2$ would be defined. One must use the boundary functional (15) with this aim.

Let's consider the case $n=0, k=0$. The system of the movement equations (13) simplifies to one equation $\left(v_{00}=0\right)$

$$
r^{2} u_{00}(r)+r u_{00}^{\prime}(r)+q^{2} r^{2} u_{00}(r)-u_{00}(r)=\frac{\mu_{0}-1}{\mu} r \delta_{0}^{\prime}(r), 0<r<a
$$

with the boundary conditions

$$
a u_{00}^{\prime}(a)+\bar{\mu} u_{00}(a)=\bar{\mu} a \delta_{0}(a)
$$

This problem has a simple solution in the Bessel functions [40].

3.2 General case of the initial boundary problem transformation

The new unknown functions associated with the original ones by the correspondences

$$
W_{n k}^{(1)}(r)=u_{n k}(r)+v_{n k}(r), W_{n k}^{(2)}(r)=u_{n k}(r)-v_{n k}(r), W_{n k}^{(3)}(r)=w_{n k}(r)
$$

should be input before considering of the general case for the Fourier transformation parameters' values $n \geq 1, k \geq 1$. As a result of the transformations, the system (13) takes the form

$$
\begin{gathered}
{\left[\kappa\left(D_{r}-(\alpha+1)^{2} r^{-2}\right)-(\kappa-1) \beta^{2}+q^{2}\right] W_{n k}^{(1)}(r)+} \\
+\left[D_{r}-2 \alpha r^{-1} \partial_{r}+\left(\alpha^{3}-1\right) r^{-2}\right] W_{n k}^{(2)}(r)+ \\
+2 \beta\left(\partial_{r}-\alpha r^{-1}\right) W_{n k}^{(3)}(r)=4 \nu \delta_{n}^{\prime}(r), \\
{\left[D_{r}+2 \alpha r^{-1} \partial_{r}+\left(\alpha^{2}-1\right) r^{-1}\right] W_{n k}^{(1)}(r)+} \\
+\left[\kappa\left(D_{r}-(\alpha+1)^{2} r^{-2}\right)-(\kappa-1) \beta^{2}+q^{2}\right] W_{n k}^{(2)}(r)+ \\
+2 \beta\left(\partial_{r}+\alpha r^{-1}\right) W_{n k}^{(3)}(r)=4 \nu \delta_{n}^{+}(r), \\
-\beta\left(\partial_{r}+(\alpha+1) r^{-1}\right) W_{n k}^{(1)}(r)-\beta\left(\partial_{r}-(\alpha-1) r^{-1}\right) W_{n k}^{(2)}(r)+ \\
+\left[(\kappa-1)\left(D_{r}-\alpha^{2} r^{-2}\right)\right. \\
\left.-(\kappa+1) \beta^{2}+q^{2}\right] W_{n k}^{(3)}(r)=4(1-\nu) \beta \delta_{n}(r),
\end{gathered}
$$

where $\kappa=3-4 \nu, \delta_{n}^{\mp}(r)=\delta_{n}(r) \mp \alpha r^{-1} \delta_{n}(r)$. The boundary conditions (14) are transformed by analogical transformations, and are written with the help of the newly input unknown functions

$$
\begin{gathered}
a\left[W_{n k}^{(1) \prime}(a)+W_{n k}^{(2) \prime}(a)\right]+\bar{\mu}\left[(\alpha+1) W_{n k}^{(1)}(a)+\right. \\
\left.+(1-\alpha) W_{n k}^{(2)}(a)+2 \beta a W_{n k}^{(3)}(a)\right]=2 \bar{\mu} a \delta_{n}(a), \\
a\left[W_{n k}^{(1) \prime}(a)-W_{n k}^{(2) \prime}(a)\right]+\left[-(\alpha+1) W_{n k}^{(1)}(a)+(1-\alpha) W_{n k}^{(2)}(a)\right]=0, \\
+2 W_{n k}^{(3) \prime}(a)-\beta\left[W_{n k}^{(1)}(a)-W_{n k}^{(2)}(a)\right]=0,
\end{gathered}
$$


Let's formulate the vector boundary problem for the equations system (20) and boundary conditions (21). The matrix differential operator, the vectors and the additional matrix are given as

$$
L_{n k} \mathbf{W}(r)=\mathbf{F}(r), 0<r<a
$$

where

$$
\begin{gathered}
L_{n k}=\left(\begin{array}{c}
a_{11}, a_{12}, a_{13} \\
a_{21}, a_{22}, a_{23} \\
a_{31}, a_{32}, a_{33}
\end{array}\right) \\
a_{11}=\kappa\left(D_{r}-(\alpha+1)^{2} r^{-2}\right)-(\kappa-1) \beta^{2}+q^{2}, a_{12}=D_{r}-2 \alpha r^{-1} \partial_{r}+\left(\alpha^{2}-1\right) r^{-2}, \\
a_{13}=2 \beta\left(\partial_{r}-\alpha r^{-1}\right), a_{21}=D_{r}+2 \alpha r^{-1} \partial_{r}+\left(\alpha^{2}-1\right) r^{-2}, \\
a_{22}=\kappa\left(D_{r}-(\alpha-1)^{2} r^{-2}\right)-(\kappa-1) \beta^{2}+q^{2}, a_{23}=2 \beta\left(\partial_{r}+\alpha r^{-1}\right), \\
a_{31}=-\beta\left(\partial_{r}+(\alpha+1) r^{-1}\right), a_{32}=-\beta\left(\partial_{r}-(\alpha-1) r^{-1}\right), \\
a_{33}=(\kappa-1)\left(D_{r}-\alpha^{2} r^{-2}\right)-(\kappa+1) \beta^{2}+q^{2} \\
\mathbf{W}(r)=\left(\begin{array}{c}
W_{n k}^{(1)}(r) \\
W_{n k}^{(2)}(r) \\
W_{n k}^{(3)}(r)
\end{array}\right), \mathbf{F}(r)=\left(\begin{array}{c}
4 \mu \delta_{n}^{-}(r) \\
4 \mu \delta_{n}^{+}(r) \\
-4 \beta(1-\mu) \delta_{n}(r)
\end{array}\right)
\end{gathered}
$$

In this case matrix $H$ should be taken in the form

$$
\mathbf{H}_{n}(r, s)=\left(\begin{array}{c}
J_{\alpha+1}(r s), 0,0 \\
0, J_{\alpha-1}(r s), 0 \\
0,0, J_{\alpha}(r s)
\end{array}\right)
$$

\section{The solution of the vector boundary problem}

A solution of the inhomogeneous vector equation is constructed as the superposition of the homogeneous equation's (22) general solution $\mathbf{W}_{0, k}(r)$ and the inhomogeneous equation's particular solution $\mathbf{W}_{* k}(r)$. One has to solve the matrix homogenous equation $L_{n k}(\mathbf{W}(r))=0,0<r<a$ [21] to obtain the vector solution $\mathbf{W}_{0}(r)$. The choosing of matrix $\mathbf{H}_{n}(r, s)$ in the form (23) provides the correspondence validity $L_{n k} \mathbf{H}_{n}(r, s)=-\mathbf{H}_{n}(r, s) \mathbf{M}_{k}(s)$. It can be proved obviously by direct checking, where

$$
\begin{gathered}
\mathbf{M}_{k}(s)=\left(\begin{array}{c}
\kappa s^{2}+(\kappa-1) \beta^{2}-q^{2},-s^{2}, 2 \beta s \\
-s^{2}, \kappa s^{2}+(\kappa-1) \beta^{2}-q^{2},-2 \beta s \\
\beta s,-\beta s,(\kappa-1) s^{2}+(\kappa+1) \beta^{2}-q^{2}
\end{array}\right) \\
\mathbf{M}_{k}^{-1}(s)=\frac{1}{\Delta(\beta, s)}\left(\begin{array}{c}
\kappa s^{2}+(\kappa-1) \beta^{2}-q^{2}, s^{2},-2 \beta s \\
s^{2}, \kappa s^{2}+(\kappa-1) \beta^{2}-q^{2}, 2 \beta s \\
-\beta s, \beta s,(\kappa-1) s^{2}+(\kappa+1) \beta^{2}-q^{2}
\end{array}\right) \\
\Delta(\beta, s)=\prod_{i=1}^{4}\left(s-s_{i}\right), s_{1,2}= \pm \frac{\sqrt{\left(q^{2}-(\kappa+1) \beta^{2}\right)}}{\sqrt{(\kappa+1)}}, s_{3,4}= \pm \frac{\sqrt{\left(q^{2}-(\kappa-1) \beta^{2}\right)}}{\sqrt{(\kappa-1)}}
\end{gathered}
$$

As with the previous case, one needs to calculate the residual at one of the poles, for example at the pole $s_{1}$, to construct matrix solution with the help of the formula $\mathbf{Y}(r)=\frac{1}{2 \pi i} \oint_{C} \mathbf{H}_{n}(r, s) \mathbf{M}_{k}^{-1}(s) d s($ is the closed contour, covering one of the inverse matrix's pole). The structure of the obtained matrix $\mathbf{Y}(r)$ is

$$
\begin{gathered}
\mathbf{Y}(r)= \\
=\left(\begin{array}{c}
i I_{\alpha+1}\left(r s_{*}\right)\left((\kappa-1) q^{2}+\left(1-\kappa^{2}\right) \beta^{2}\right),-i I_{\alpha_{1}}\left(r s_{*}\right) s_{*}^{2},-2 I_{\alpha+1}\left(r s_{*}\right) \beta s_{*} \\
i I_{\alpha-1}\left(r s_{*}\right) s_{*}^{2}, i I_{\alpha-1}\left(r s_{*}\right)\left((\kappa-1) q^{2}+\left(1-\kappa^{2}\right) \beta^{2}\right), 2 I_{\alpha-1}\left(r s_{*}\right) \beta s_{*} \\
-i I_{\alpha}\left(r s_{*}\right) \beta s_{*}, i I_{\alpha}\left(r s_{*}\right) \beta s_{*}, i I_{\alpha}\left(r s_{*}\right)\left(\kappa q^{2}-(\kappa-1)(\kappa+1)^{2}\right) \\
s_{*}=\sqrt{(\kappa+1) \beta^{2}-q^{2}}
\end{array}\right)
\end{gathered}
$$


To provide the obtained values' realness, the general solution regular at zero is constructed in the form $\mathbf{W}_{0 k}(r)=\mathbf{Y}(r)\left(i C_{1}, i C_{2}, C_{3}\right)^{T},\left(C_{i}, i=1,2,3\right.$ are the arbitrary real constants). As a result, the formulas for the displacements' transformations are obtained

$$
\begin{gathered}
W_{0, n k}^{1}(r)=C_{1} I_{\alpha+1}\left(r s_{*}\right)\left((\kappa-1) q^{2}+\left(1-\kappa^{2}\right) \beta^{2}\right)- \\
-C_{2} I_{\alpha+1}\left(r s_{*}\right) s_{*}^{2}-2 C_{3} I_{\alpha+1}\left(r s_{*}\right) \beta s_{*} \\
W_{0, n k}^{2}(r)=C_{1} I_{\alpha-1}\left(r s_{*}\right) s_{*}^{2}+C_{2} I_{\alpha-1}\left((\kappa-1) q^{2}+\left(1-\kappa^{2}\right) \beta^{2}\right)+ \\
+2 C_{3} I_{\alpha-1}\left(r s_{*}\right) \beta s_{*} \\
W_{0, n k}^{3}(r)=-C_{1} I_{\alpha}\left(r s_{*}\right) \beta s_{*}+C_{2} I_{\alpha}\left(r s_{*}\right) \beta s_{*}- \\
-C_{3} I_{\alpha}\left(r s_{*}\right)\left(\kappa q^{2}-(\kappa-1)(\kappa+1)^{2}\right), \\
s_{*}=\sqrt{(\kappa+1) \beta^{2}-q^{2}}
\end{gathered}
$$

Now let's find the particular solution. The right hand part of the equation (22) is extended on the whole axis by the zero at the segment $r>a$ :

$$
\mathbf{L}_{n k} \mathbf{W}_{* k}(r)=\mathbf{F}^{+}(r), 0<r<\infty \text {. }
$$

The matrix integral transformation with kernel $\mathbf{H}_{n}(r, s)$, described by formulas (23), is applied to the equation (25). The particular solution in the transformation's domain will take the form $\mathbf{W}_{s}=$ $-\mathbf{M}_{n k}^{-1}(s) \mathbf{F}_{s}^{+}, \mathbf{F}_{s}^{+}=\int_{0}^{a} \rho \mathbf{H}_{n}(\rho, s) \mathbf{F}(\rho) d \rho$. The vector's original formula is written with the help of the fundamental matrix [38] after inversion

$$
\begin{aligned}
& \mathbf{W}_{s}(r)=-\int_{0}^{a} \Phi_{n k}(r, \rho) \mathbf{F}(\rho) d \rho, 0<r<a, \\
& \Phi(r, \rho)=-\int_{0}^{\infty} s \mathbf{H}_{n}(r, s) \mathbf{M}_{n}^{-1}(s) \mathbf{H}_{n}(\rho, s) d s
\end{aligned}
$$

the elements of this matrix are the integrals of (17) type:

$$
\begin{gathered}
\Phi(r, \rho)= \\
=\left(\begin{array}{c}
\kappa \Omega_{\alpha+1, \alpha+1}^{3}+\left((\kappa+1) \beta^{2}+q^{2}\right) \Omega_{\alpha+1, \alpha+1}^{1}, \kappa \Omega_{\alpha+1, \alpha+1}^{3},-2 \beta \Omega_{\alpha+1, \alpha}^{2} \\
\Omega_{\alpha+1, \alpha-1}^{3}, \kappa \Omega_{\alpha-1, \alpha-1}^{3}+\left((\kappa+1) \beta^{2}-q^{2}\right) \Omega_{\alpha-1, \alpha-1}^{1}, 2 \beta \Omega_{\alpha-1, \alpha}^{2} \\
-\beta \Omega_{\alpha+1, \alpha}^{2}, \beta \Omega_{\alpha-1, \alpha}^{2},(\kappa+1) \Omega_{\alpha, \alpha}^{3}+\left((\kappa-1) \beta^{2}-q^{2}\right) \Omega_{\alpha, \alpha}^{1}
\end{array}\right) \\
A^{2}=\beta^{2}-\frac{q^{2}}{\kappa+1}, B^{2}=\beta^{2}+\frac{q^{2}}{\kappa-1}
\end{gathered}
$$

With the help of the formula $\mathbf{W}_{* k}(r)=\int_{0}^{a} \Phi(r, \rho) \mathbf{F}_{n k}^{+}(\rho) d \rho$, the partial solution of the vector equation is obtained.

$$
\begin{gathered}
W_{*, n k}^{1}(r)=4 \nu \int_{0}^{a}\left(\kappa \Omega_{\alpha+1, \alpha+1}^{3}(r, \rho)+\left((\kappa+1) \beta^{2}+q^{2}\right) \Omega_{\alpha+1, \alpha+1}^{1}(r, \rho)\right) \delta_{n}^{-}(\rho) d \rho+ \\
+4 \nu \int_{0}^{a} \kappa \Omega_{\alpha+1, \alpha-1}^{3}(r, \rho) \delta_{n}^{+}(\rho) d \rho+8 \beta^{2}(1-\nu) \int_{0}^{a} \Omega_{\alpha+1, \alpha}^{2}(r, \rho) \delta_{n}(\rho) d \rho \\
W_{*, n k}^{2}(r)=4 \nu \int_{0}^{a} \Omega_{\alpha+1, \alpha-1}^{3}(r, \rho) \delta_{n}^{-}(\rho) d \rho+ \\
4 \nu \int_{0}^{a}\left(\kappa \Omega_{\alpha-1, \alpha-1}^{3}(r, \rho)+\left((\kappa+1) \beta^{2}-q^{2}\right) \Omega_{\alpha-1, \alpha-1}^{1}(r, \rho)\right) \delta_{n}^{+}(\rho) d \rho- \\
-8 \beta^{2}(1-\nu) \int_{0}^{a} \Omega_{\alpha-1, \alpha}^{2}(r, \rho) \delta_{n}(\rho) d \rho \\
W_{*, n k}^{3}(r)=-4 \nu \beta \int_{0}^{a} \Omega_{\alpha+1, \alpha}^{2}(r, \rho) \delta_{n}^{-}(\rho) d \rho+4 \nu \beta \int_{0}^{a} \Omega_{\alpha-1, \alpha}^{2}(r, \rho) \delta_{n}^{+}(\rho) d \rho- \\
-4 \beta(1-\nu) \int_{0}^{a}\left((\kappa+1) \Omega_{\alpha, \alpha}^{3}(r, \rho)+\left((\kappa-1) \beta^{2}-q^{2}\right) \Omega_{\alpha, \alpha}^{1}(r, \rho)\right) \delta_{n}(\rho) d \rho
\end{gathered}
$$

The sum of the general regular solution (24) and partial solution (26) will finally define the three input unknown functions, if three arbitrary constants in this sum will be found, one has to use the 
boundary conditions (21) for it. The transformations of the initial displacements one has to find by the formulas $\left\|\begin{array}{l}u_{n k}(r) \\ v_{n k}(r)\end{array}\right\|=\frac{W_{n k}^{(1)}(r)+W_{n k}^{(2)}(r)}{2}, w_{n k}(r)=W_{n k}^{(3)}(r)$.

The application of the inverse Fourier's transformation allows us to write the final representations

$$
\begin{gathered}
\tilde{u}(r, \varphi, z)=\frac{2}{h} \sum_{k=1}^{\infty} u_{0 k}(r) \cos \beta_{k} z+\frac{4}{r h} \sum_{n=1}^{\infty} \sum_{k=0}^{\infty} u_{n k}(r) \cos \alpha_{n} \varphi \cos \beta_{k} z \\
\tilde{v}(r, \varphi, z)=\frac{4}{r h} \sum_{n=1}^{\infty} \sum_{k=0}^{\infty} v_{n k}(r) \cos \alpha_{n} \varphi \cos \beta_{k} z \\
\tilde{w}(r, \varphi, z)=\frac{2}{r h} \sum_{k=1}^{\infty} w_{0 k}(r) \sin \beta_{k} z+\frac{4}{r h} \sum_{n=1}^{\infty} \sum_{k=0}^{\infty} w_{n k}(r) \cos \alpha_{n} \varphi \sin \beta_{k} z .
\end{gathered}
$$

The obtained series are conditionally convergent, this is connected with application of the integral transformation with regard to the variable $z$ by the inhomogeneous boundary conditions (the conditionally convergence of the series obtained in such cases is proved in [20]). This is why before the calculations of the stress values and differentiation of the expressions of the displacements, one needs to extract a weak convergent part of the series. To do this the following procedure is proposed: a series is separated on the sum of the two summands $\left(\sum_{k=1}^{N}+\sum_{k=N+1}^{\infty}\right) a_{k}(r)$. The sum $\sum_{k=1}^{N} \tilde{a}_{k}$ is added and subtracted from this expression, (here $\tilde{a}_{k}$ are asymptotical expressions of the series' general terms, when $k \rightarrow \infty$; a general term of the series is changed by an asymptotical expression at the second summand $\sum_{k=1}^{\infty} a_{k}(r)=\sum_{k=1}^{\infty} \tilde{a}_{k}(r)+\sum_{k=1}^{N} a_{k}(r)-\sum_{k=1}^{N} \tilde{a}_{k}(r)$. The obtained series in this equality is summarized with the known formulas [39].

The series in this form are substituted in the formulas for the stress calculation. The formulas (27) will determine the wave field of the elastic wedge-liked plate, if the constants $\delta, A$ are known (these parameters determine the value of the plate's face subsidence). The formulas for the stress $\sigma_{z}(r, \varphi, z, t)$ and displacement $u_{z}(r, \varphi, z, t)$ are calculated and substituted into the rigid plate's movement equations (4). The system of the two equations is obtained and solved for the estimation of the unknown constants $\delta, A$. It finishes the construction of the wedge-like plate's wave field.

It should be noted, that the proposed statement of the problem implies, that friction is absent at the bottom face of the plate, and displacements $u_{z}(r, \varphi, z, t)$ through the axis $0 z$ equals zero. Such boundary conditions mean that at certain frequency values, separation of the bottom face can occur. In order to find out whether it is possible to load the plate and to eliminate the possibility of base separation, we take into account the weight of plate and find out whether it exceeds the dynamic stresses, in this case, the plate's stresses caused by its own weight.

In a static statement, the problem of the stress state of the elastic wedge plate under its own weight has been solved earlier and an elementary solution was obtained [23]

$$
\begin{gathered}
u(r, \varphi, z)=v(r, \varphi, z)=0, w(r, \varphi, z)=\left(G \mu_{*}\right)^{-1} \gamma z(0.5 z-h) \\
\sigma(r, \varphi, z)=\gamma(z-h)
\end{gathered}
$$

assuming the conditions of ideal contact on the bases and at the edge $(\gamma$ is the dead weight of the plate material). (The correctness of the solution can be verified by direct substitution of it in the movement equations and boundary conditions).

To use this result with the proposed problem one has to change the boundary conditions at the edge with the conditions of ideal contact $u_{r}(a, \varphi, z, t)=0, \tau_{r \varphi}(a, \varphi, z, t)=0, \tau_{r z}(a, \varphi, z, t)=0$. The general scheme of the solution remains the same, except for the one-dimensional problem where the boundary conditions (6) will be changed (with their help one has to determine the unknown constants of the general solution).

\section{The results of the numerical analyses}

The main goal of the numerical investigation in the proposed research is the identification of the elastic wedge plate eigenfrequencies. The oscillations of the finite steel plate radius $r=a$ with an angle of 
the opening $\varphi=\Upsilon$ and height $h$ are investigated. The transition to the dimensionless coordinates was done

$$
\Omega=\frac{2 \Upsilon h}{\pi c_{2}}, R=\frac{a}{h}, \bar{u}_{r}=\frac{u_{r}}{h}, \bar{u}_{\varphi}=\frac{u_{\varphi}}{h}, \bar{u}_{z}=\frac{u_{z}}{h}, \sigma=\frac{\sigma_{z}}{h}
$$

The oscillation's eigenfrequencies were found by the following algorithm. The displacements and stresses values were obtained by a given load in the same points of the region for different values of frequencies, which previously were taken with step 0.1 . In the case where a transition through the eigenfrequency was noted, it was also noted that phases of the wave field calculated components are changed. After that, the range of frequencies, during the transition through which was noted this phenomenon, was divided by a smaller step - 0.01 - in order to clarify the value of the eigenfrequency - and then again the procedure of displacement and stress calculations for a given interval of the frequencies is used. The clarification of the frequencies' values was produced to the fourth decimal figure. This method of searching eigenfrequencies apparently has was first proposed and tested in [32].

The eigenfrequencies $\Omega$ were investigated and the dependence between their values and plate's geometrical parameters was established.

In Fig. 2 and Fig. 3 the changes in the spectrum of the wedge plate's eigenfrequencies $\Omega$ depending on changes in the relative radius $R=\frac{a}{h}$ and the angle of wedge plate's opening $\Upsilon$ are given.

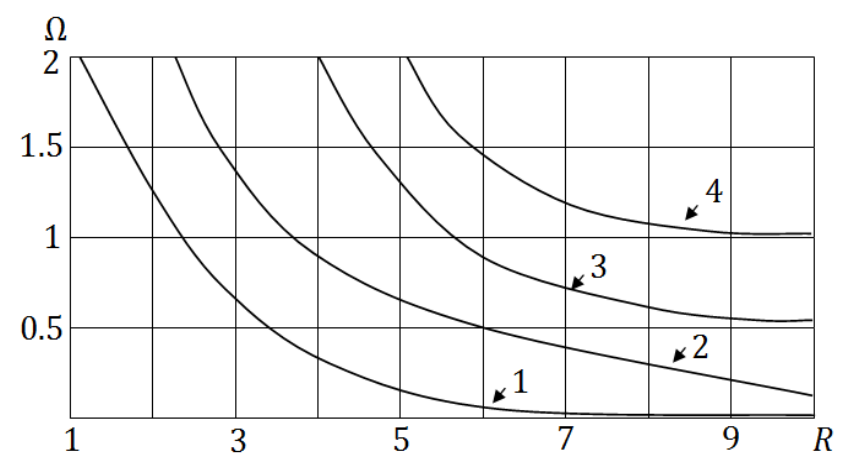

Fig. 2 The changes in the spectrum on changes of the relative radius $R=\frac{a}{h}$

As can be seen on the graphs, the value of the eigenfrequencies in this case (the curvature in the graphs corresponds to the number of an eigenfrequency) is inversely proportional to the relative radius (the opening angle corresponds to the value $\Upsilon=\frac{\pi}{4}$ ), and to the angle of the wedge plate's opening (the relative radius was chosen $R=4$ ).

Comparison of the obtained results with the results shown in [15] was conducted. It confirmed the inverse dependence of the frequencies as from the dimensionless radius so and from the elastic plate's opening angle.

A more complicated picture of the eigenfrequencies $\Omega$ distribution is observed when an angle of the wedge is smaller than $\Upsilon=\frac{\pi}{4}$. As seen on Fig. 4 (opening angle $\Upsilon=\frac{\pi}{12}$ ), by increasing the dimensionless radius $3 \leq R \leq 7$, the segments appear where the eigenfrequency is constant. These segments lie in the frequency range $1.55<\tilde{\omega}<1.56$. As described in detail in [16], the resonance phenomenon corresponds to such segments. For the frequencies below the left edge of this segment, as in the previous case, the inverse dependence of the eigenfrequency form of the wedge-like plate is noted.

With the aim of verifying this fact, the displacement and stress for various Eigenforms were calculated. In particular, Figure 5 shows the dominant displacement $\bar{u}_{z}$ at the line $R=1, \varphi=\frac{\Upsilon}{2}, z=\frac{1}{2}$ corresponding to the frequencies that lie to the left edge of the resonance frequency (lines 1 and 2 of Fig. 5) and to the frequency which is a mid-range $1.55<\tilde{\omega}<1.56$ (line 3 in Fig. 5). As can be seen, in the last case, the displacements' values increase sharply as it approaches at the edge of the plate 


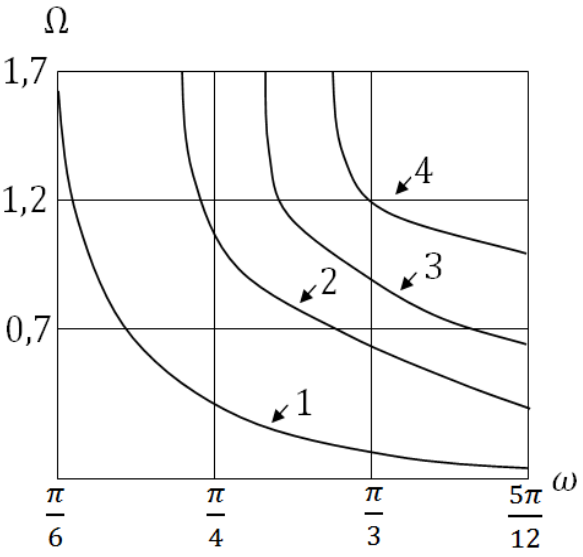

Fig. 3 The changes in the spectrum on changes of the angle of wedge opening $\Upsilon$

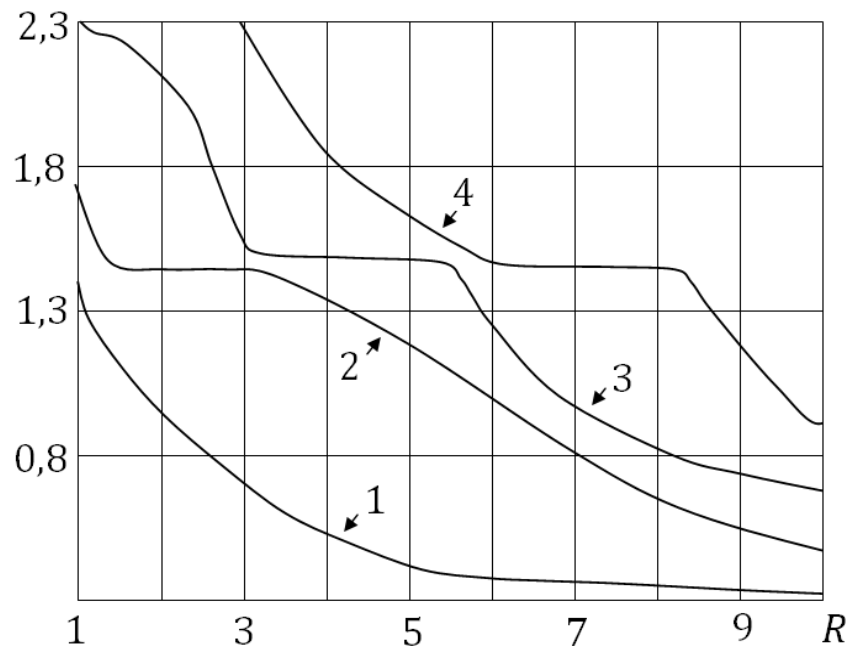

Fig. 4 The changes in the spectrum when angle of wedge is smaller $\Upsilon=\frac{\pi}{4}$

$R=1$, and the contact mode has a characteristic form. A similar pattern is observed for the stress (Fig. 6).

Here the stress values are shown corresponding to the frequencies that lie to the left edge of the resonance frequency (lines 1 and 2) and to the frequency which is a mid-range $1.55<\tilde{\omega}<1.56$ (line $3)$.

The graphs of the displacements $\bar{u}_{r}, \bar{u}_{\varphi}$ are shown at Fig. 7 , Fig. 8 correspondently. It can be seen that the behaviour of the displacements during the pass through the resonance frequency is similar. Also it can be noticed that the absolute value of these displacements is significantly less than the values of normal displacement $\bar{u}_{z}$. (Authors would like to underline that the behaviour of the mechanical characteristics at the vertex of the three-edged angle was not investigated).

Table. 1 presents data showing the dependence of the edge resonance's frequency on the dimensionless radius of the plate (the angle of the wedge's opening $\Upsilon=\frac{\pi}{12}$ ).

It can be noted that with an increase of the relative radius, an increase of the edge resonance's frequency is seen. The significant effect on the frequency of the plates' edge resonance has the angle 


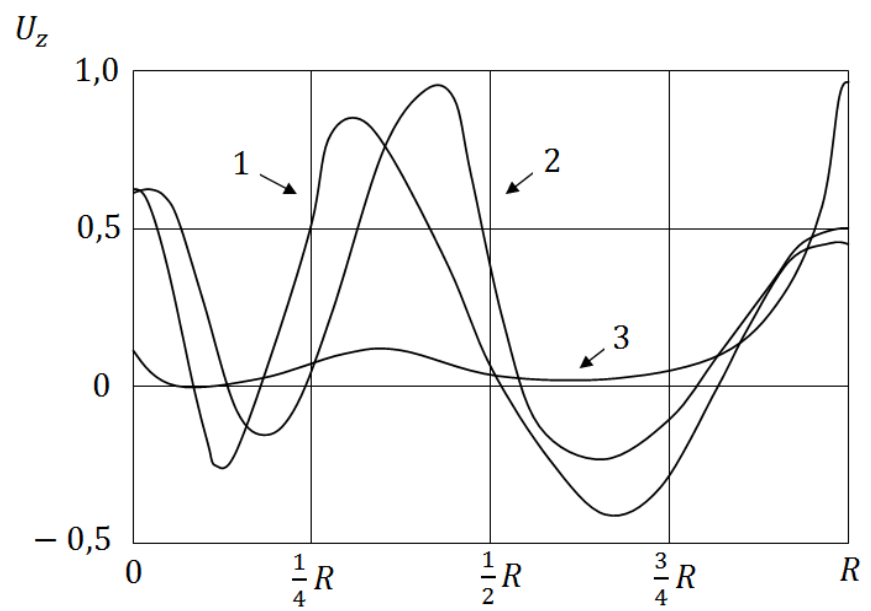

Fig. 5 The changes of dominant displacements $u_{z}$ on line $R=1, \varphi=\frac{r}{2}, z=\frac{1}{2}$

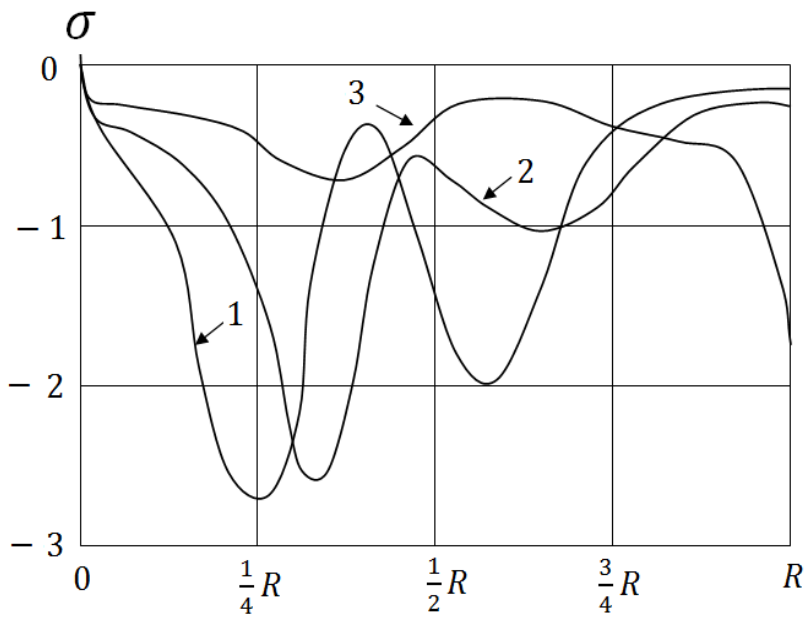

Fig. 6 The changes of stress on line $R=1, \varphi=\frac{r}{2}, z=\frac{1}{2}$

of the plate's opening. Analysis of the results (shown in Table. 2 for the dimensionless radius $R=4$ ) shows that increasing of the opening angle's values of the wedge plate lead to an increase of the edge resonance frequency values.

It was established that increasing of Poisson's coefficient values leads to the increasing of edge resonance frequency also.

Also it was investigated at what frequency of forced oscillations the separation of the base begins, Such effect can be observed when the conditions of the ideal contact are given at the base of the plate. Calculations showed that with values of the forced oscillations' frequency $\tilde{\omega}<2.62$, the separation of the plate base's points with an opening angle $\Upsilon=\pi / 3$ is not observed. A significant impact on the value of the frequency separation is the angle of the wedge opening. Thus, with the opening angle of the plate $\Upsilon=\pi / 4$ the separation don't start till the frequency $\tilde{\omega}=1.96$, and with the angle $\Upsilon=\pi / 6$ the separation was not found till the frequency $\tilde{\omega}<1.65$.

To validate the calculations the values of the static stress $\sigma_{z}(r, \varphi, z)$ on the bottom face in the case of the wedge plate loading by its dead weight (at the edge $r=a$ are assumed the conditions of ideal 


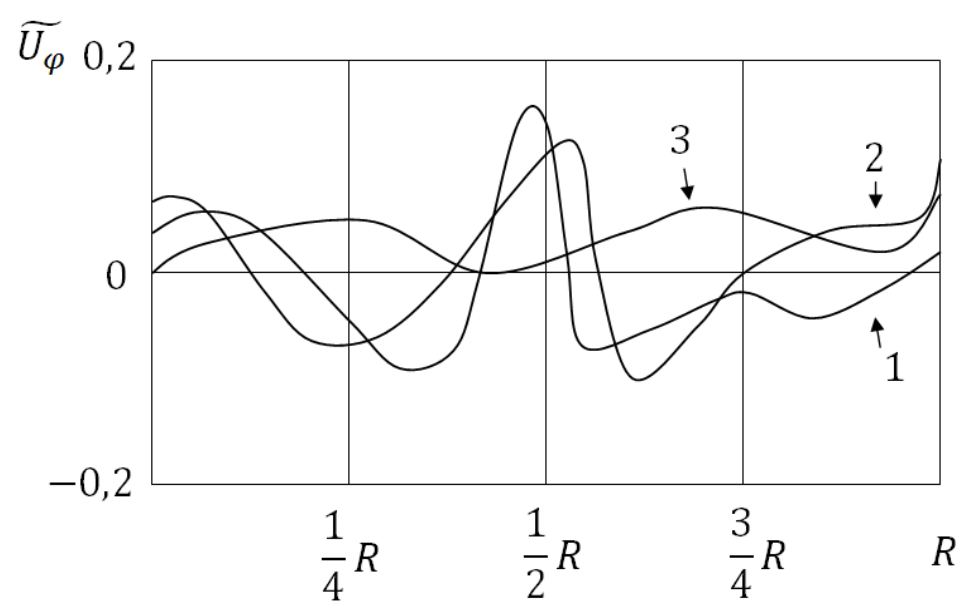

Fig. 7 The changes of displacement $u_{\varphi}$ on line $R=1, \varphi=\frac{r}{2}, z=\frac{1}{2}$

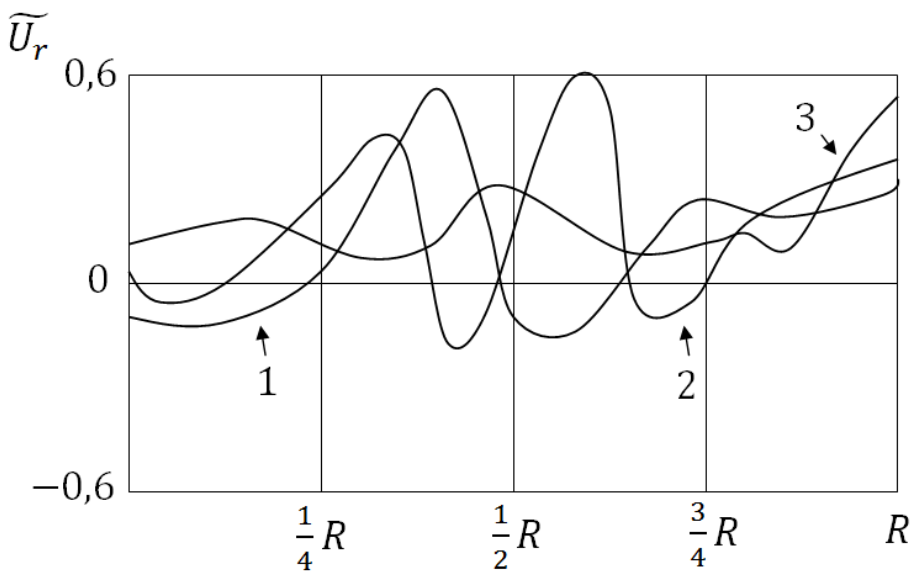

Fig. 8 The changes of displacement $u_{r}$ on line $R=1, \varphi=\frac{r}{2}, z=\frac{1}{2}$

Table 1 The dependence of the edge resonance's frequency on the dimensionless radius of the plate

\begin{tabular}{|l|c|}
\hline$R=3$ & $\tilde{\omega} \in$ \\
& $(1.551 ; 1.552)$ \\
\hline$R=5$ & $\tilde{\omega} \in$ \\
& $(1.555 ; 1.556)$ \\
\hline$R=7$ & $\tilde{\omega} \in$ \\
& $(1.555 ; 1.556)$ \\
\hline$R=12$ & $\tilde{\omega} \in$ \\
& $(1.558 ; 1.559)$ \\
\hline$R=15$ & $\tilde{\omega} \in$ \\
& $(1.621 ; 1.622)$ \\
\hline
\end{tabular}

contact) were calculated [23].The results correspond to line 1 at Fig. 9. These stresses were compared with the values of the dynamic stresses $\sigma_{z}(r, \varphi, z, t)$ that arise in a similar problem when the upper face is uploaded with the oscillating force through an absolutely rigid overlay. At the Fig.9 the line 2 corresponds to the dynamic stresses that with values of the forced oscillations' frequency $\tilde{\omega}=0.05$. As it seen, the graphics coincide close enough. Calculations of the dynamic stress with forced oscillations' 
Table 2 The dependence of the edge resonance's frequency on the plate's opening angle

\begin{tabular}{|l|l|l|}
\hline$\Upsilon=\pi / 12$ & $\Upsilon=\pi / 6$ & $\Upsilon=\pi / 4$ \\
\hline $1.554<\tilde{\omega}<1.555$ & $1.555<\tilde{\omega}<1.556$ & $1.556<\tilde{\omega}<1.557$ \\
\hline
\end{tabular}

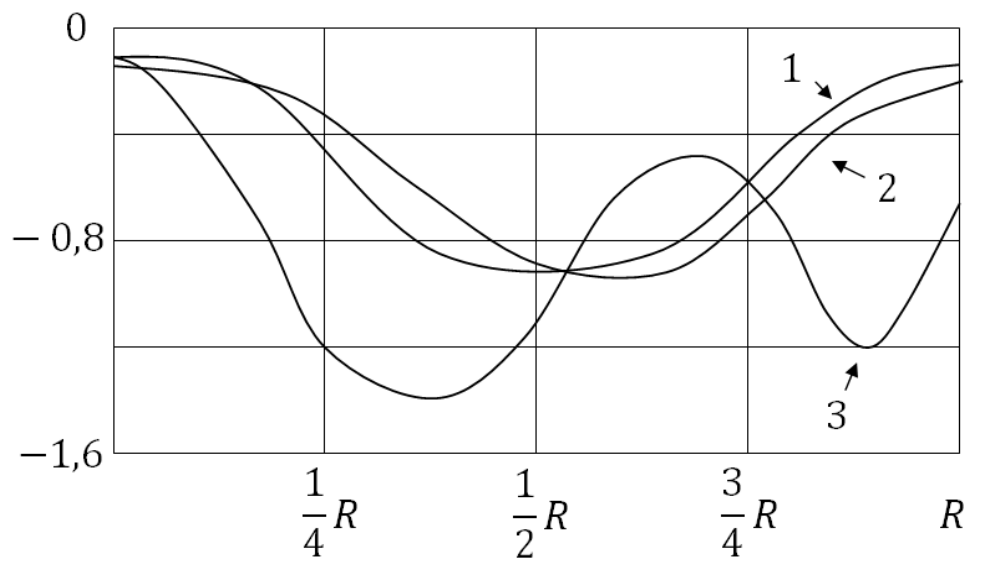

Fig. 9 The stresses for the dynamic and static cases

frequency $\tilde{\omega}=1.5$ showed that values of dynamic stresses are practically twice the value of static stresses (line 3 on Fig. 9).

\section{Conclusions}

The exact solution of the problem on the forced oscillations of an elastic wedge shaped thick finite plate is derived. Numerical results obtained on the basis of exact solution formulas of the problem can be used to solve similar problems by the approximate or numerical methods.

The edge resonance frequencies and the influence of the geometrical parameters of the wedge plate were studied. The frequency at which the lower edge separates from the base is identified, and its dependence on the plate opening angle's value is studied.

Important mechanical laws are established during the numerical analyses:

1) It was indicated that an inverse dependence of eigenfrequency values on linear dimension value and opening angle of a plate by the angles larger than $\pi / 4$.

2) It was noted the edge resonance phenomenon for the values of an opening angle smaller than $\pi / 4$. It is established that with increasing values of the linear size and the opening angle the edge resonance first frequency value increases also.

3) The conditions for the appearance of tensile stresses were studied in the case of smooth contact conditions on the lower surface of the plate. It was found that a significant impact on their appearance is an opening angle value - with its increasing tensile stress occurring at higher frequencies. When values of the angle opening are greater than $\pi / 3$ the separation of the base is not observed.

The proposed method of the solution, based on special transformations of the movement equations and the subsequent application of the matrix integral transformations method allows the solution of the dynamic problem of the forced oscillations of an elastic wedge shaped infinite thick plate.

Acknowledgements The research is supported by the British Council Ukraine - International high education (Ref. UKR16EG/28/29.01.16). Gratitude for Simon Dyke for the editing of the articles text. 


\section{References}

1. Hu, Y., Wang, K., 2006. Bending like behavior of then wedge-shaped elastic fault blocks. J. Geophys. Res. 111, B06409, DOI:10.1029/2005JB003987.

2. Poruchikov, V., 1993. Methods of the Classical Theory of Elastodynamics (Solution to Three-Dimensional Elastodynamic Problems with Mixed Boundary Conditions for Wedge-Shaped Domains), Springer Berlin Heidelberg, pp. 190-219.

3. Grinchenko, V., 1978. The equilibrium and steady-state oscillations of the elastic bodies (in Russian), Naukova dumka, Kiev.

4. Starovoytov, E., Kubenko, V., Tarlakovsky, D., 2009. The oscillations of the roung threelayered plates connected with the elastic base (in Russian). Izv. Vuzov. Aviacionnaya technica. 2, 16-39.

5. Bert, C.W., 1991. Research on dynamic behavior of composite and sandwich plates. Y: part I. Shock Vibr. Digest. 23(6), 3-14.

6. Vorovich, I., Babeshko, V., 1979. The dynamic mixed elasticity problems for the nonclassical ranges (in Russian), Nauka, Moscow.

7. Vatulyan, A., Chebakova, E., 2004. The fundamental solutions for the orthtropic elastic medium in the case of the steady state oscillations. Journal of Applied Mechanics and Technical Physics. 5, 131-39.

8. Menshykova, M.V., Menshykov, O.V., Guz, I.A., Wuensche, M., Zhang, C., 2016. A boundary integral equation method in the frequency domain for cracks under transient loading. Acta Mechanica. 227(11), 3305-3314.

9. Guz, A.N., Guz, I.A., Menshykov, A.V., Menshykov, V.A., 2013. Three-dimensional problems in the dynamic fracture mechanics of materials with interface cracks (review). International Applied Mechanics. 49(1), 1-61.

10. Kit H.S., Mykhas'kiv V.V., Khaj O.M., 2002. Analysis of the steady oscillations of a plane absolutely rigid inclusion in a three-dimensional elastic body by the boundary element method. Journal of Applied Mathematics and Mechanics. 66(5), 817-824

11. Astitva Tripathi, Anil K. Bajaj, 2016. Topology optimization and internal resonances in transverse vibrations of hyperelastic plates. International Journal of Solids and Structures. 81, 311-328.

12. Shaban, M., Alipour, M.M., 2011. Semi-analytical solution for free vibration of thick functionally graded plates rested on elastic foundation with elastically restrained edge. Acta Mechanica Solida Sinica. 24(4), 340-354.

13. Wang, G., Wereley, N.M., 2002. Free in-plane vibration of rectangular plates. AIAA J. 40(5), 953959.

14. Hasheminejad, S.M., Ghaheri, A., Rezaei, S., 2012. Semi-analytic solutions for the free in-plane vibrations of confocal annular elliptic plates with elastically restrained edges. J. Sound. Vib. 331(2), 434456.

15. Liew, K.M., Hung, K.C., Lim, M.K., 1993. A continuum three-dimensional vibration analysis of thick rectangular plates. International Journal of Solids and Structures. 30(24), 3357-3379.

16. Lee, W.M., Chen, J.T., 2011. Free vibration analysis of a circular plate with multiple circular holes by using indirect BIEM and addition theorem. J. Appl. Mech T. ASME. 78(1), 011015110.

17. Eftekhari, S., Jafari, A., 2014. Accurate variational approach for free vibration of simply supported anisotropic rectangular plates. Arch. Appl. Mech. 84(4), 607614.

18. Civalek, O., 2008. Free vibration analysis of symmetrically laminated composite plates with first-order shear deformation theory (FSDT) by discrete singular convolution method. Finite Elem. Anal. Des. 44(12), 725731 .

19. Asadi, E., Fariborz, S., 2012. Free vibration of composite plates with mixed boundary conditions based on higher-order shear deformation theory. Arch. Appl. Mech. 82(6), 755766.

20. Zhong, H.,Yu, T., 2007. Flexural vibration analysis of an eccentric annular Mindlin plate. Arch.Appl.Mech. $77(4), 185195$.

21. Chaudhuri, R.A., Xie, M., 2015. On three-dimensional asymptotic solution, and applicability of SaintVenant principle to pie-shaped wedge and end face (of a semi-infinite plate) boundary value problems. Eng. Fracture Mechanics. 142, 93-20.

22. Alexandrov, V., Pozharskii, D., 2002. The problem of an inclusion in a three-dimensional elastic wedge. Journal of Applied Mathematics and Mechanics. 66(4), 617-628.

23. Popov, G.Ya., 2001. The exact solution of the first main elasticity problem for the edgeshaped thick infinite elastic plate (in Russian). Doklady Physics. 381(6), 782-85.

24. Popov, G.Ya., Kebli, B., 2012. Exact solution of the mixed boundary value elasticity problem for an infinite wedge-shaped plate with regard for its proper weight. Journ. of Math. Sciences. 187(6), 758-771.

25. Zhou, D., Au, F.T.K., Cheung, Y.K., Lo, S.H., 2003. Three dimensional vibration analysis of circular and annular plates via the Chebyshev-Ritz method. Int. J. Solids Structures. 40(12), 30893105.

26. Houmat, A., 2004. Three dimensional hierarchical finite element free vibration analysis of annular sector plates. J. Sound Vibrations. 276(1-2), 181-193.

27. Lyapin, A., Seleznov, M., 1985. The investigation method of the steady harmonic oscillations in the composite edge-like area. Journal of Applied Mathematics and Mechanics. 49(5), 667-70.

28. Huang, C.S., McGee, O.G., Leissa, A.W., 1994. Exact analytical solutions for free vibrations of thick sectorial plates with simply supported radial edges. International Journal of Solids and Structures. 31(11), 1609-1631.

29. Xu, R.Q., 2008. Three-dimensional exact solutions for the free vibration of laminated transversely isotropic circular, annular and sectorial plates with unusual boundary conditions. Arch Appl. Mech. 78, 543-58.

30. Shi, X., Shi, D., Li, W.L., Wang, Q., 2014. A unified method for free vibration analysis of circular, annular and sector plates with arbitrary boundary conditions. J. Vib. Control. doi:10.1177/1077546314533580. 
31. Vaysfel'd, N., 2015. The exact solution of the problem on the forced oscillations of an elastic wedge shaped thick plate. Proc. of the Third Intl. Conf. Advances in Civil, Structural and Mechanical Engineering- CSM. doi: 10.15224/ 978-1-63248-062-0-31.

32. Grinchenko, V., Meleshko, V., 1981. The harmonic oscillations and waves in the elastic bodies (in Russian), Naukova Dumka, Kiev.

33. Storogev, V., Mysovsky, Yu., Sokolova, O., 2003. On particular qualities of the eigenoscillations spectrum at the transtropic disc plate near the area of the boundary resonance (in Russian). Teoreticheskaya i prikladnaya mechanics. 37, 184- 89.

34. Smoktiy, O., Moiseenko, I., 2009. The effect of the boundary resonance due the stimulation of the axysimmetrical waves in the semi-infinite transverse isotropic cylinder. Mechanics of Solids. 39, 215-23.

35. Gorodeckaya, N., Grinchenko, V., 2004. The analyses of the physical peculiarities of the boundary resonance in the elastic bodies (in Russian). Akusticheskiy vesnik. 7(1), 30-43.

36. Grantmacher, F.R., 1960. The theory of matrices, Chelsea.

37. Bateman, H., Erdelyi, A., 1955. Higher Transcendental Functions, McGraw Hill, N.Y. etc., V.2.

38. Popov, G., Abdymanapov, S., Efimov, V., 1999. The Green's functions and matrixes of the onedimensional boundary problems (in Russian), Almaty, Ruan.

39. Gradshtein, L., Rygik, L., 1963. The tables of integrals, series and products (in Russian), Nauka, Moscow.

40. Kamke, E., 1966. Handbook on differential equations in partial derivatives of the first order (in Russian), Nauka, Moscow. 